\title{
Development of Criterion of Degree of Identifiability of Parameters of Dynamic Systems
}

\author{
K. A. Neusypin, T.Y. Tsibizova, I.A. Kuznetsov \\ Department of Informatics and Control Systems, Bauman Moscow State Technical University, \\ Moscow, Russia
}

Keywords: quality of identification of parameters, algorithm of identification, criterion of identifiability.

\begin{abstract}
Criteria of definition of degree of identifiability of parameters of linear dynamic systems are investigated. On the basis of scalar approach the original numerical criterion of degree of identifiability of concrete parameters of a matrix of model of dynamic object is developed.
\end{abstract}

\section{Introduction}

The solution of tasks of control of dynamic different objects assumes use of a mathematical model of the researched process. The mathematical models received on the basis of physical or any other laws, as a rule, not always precisely reflect the researched processes in practical applications. Therefore different algorithms of identification are applied to specification of structure and parameters of a mathematical model [1,2,3] and algorithms of creation of models [4,5,6]. Algorithms of identification allow to define separate matrix parameters of model which is set a priori. As algorithms of creation of models the most exact algorithms are often used: neural networks, self-organization methods (most known method of the group accounting of arguments) and genetic algorithms. Algorithms of creation of models allow to construct model of the researched process with high accuracy, but these models are non physical [5]. Usually for the analysis and control of a number of dynamic objects it is necessary to use physical models with a priori known structure. In the course of functioning of a dynamic object in the changing conditions some parameters of models can significantly change therefore they need to be defined by algorithms of identification. Accuracy of determination of model parameters depends on properties of a matrix of model and accuracy of algorithm of identification. The choice of the used algorithm of identification is defined for reasons of opportunities of implementation in the available calculator (the special calculator, onboard central computer, terrestrial computing station, etc.) economic opportunities and features of a designed project. Precision characteristics of the most popular algorithms of identification are known [1]. The properties of matrixes of models of different dynamic objects influencing the accuracy of determination of separate parameters are studied generally only for well waste models, for example in nuclear technique, inertial navigation and some other applications. Research of specific models regarding quality of identification of parameters were carried out by practical consideration and, generally, application of such approach requires carrying out a series of difficult experiments, the long time and essential financial expenses.

In a different way solutions of the task of determination of quality of identification analytical approach - development of criterion of a measure or level of identifiability is. Development of the universal criterion of a level of identifiability allowing to calculate quality of determination of matrix parameters of model of different dynamic objects is the important task. This task acquires special relevance in case of research of the projected dynamic objects and their algorithmic support.

\section{Criteria of a level of identifiability}

Different methods of determination of a measure (level) of identifiability or a condition of definability are known [7,8,9].

The method [7], allows to define basic possibility of implementation of procedure of identification. 
The model of the linear uniform system is researched. The linear uniform system is called completely identifitsiremy on state vector $\mathrm{x}$ if in case of the given vector of initial conditions of $\mathrm{x} 0$ the matrix of parameters A can be single-digit regenerated for a finite interval of time of identification on one temporal sequence of $x=x(t)$.

Otherwise, couple $(\mathrm{A}, \mathrm{x} 0)$ is completely identified or identified quite when the set of couples $\left(A_{-}, x 0\right)$ integrated by a community of an integral curve of $x=x(t), x 0=x(0)$ degenerates in a point $A_{-}=A$. Otherwise couple isn't identified. The necessary and sufficient condition of full identifiability of couple $(\mathrm{A}, \mathrm{x} 0)$ is as follows

$$
\operatorname{Rank}\left[W_{0}\right]=\operatorname{Rank}\left[x_{0}, A x_{0}, A^{2} x_{0}, \ldots, A^{n-1} x_{0}\right] .
$$

$\mathrm{W}_{0}$ - an identifiability matrix.

The system with a unit matrix of A is not identified.

This method assumes determination of basic possibility of computation of identifiability.

The method [8] is based on computation of a condition of definability. The linear model of the signal distorted by accidental process of type a white noise is researched. Dispersion of a white noise is known and observable. The following assumptions are entered: let dispersion single, a matrix of model be steady, an input with finite energy.

The assessment of an unconditional maximum of credibility which is asimptotick an effective assessment is calculated. Quality of such estimates asymptotically doesn't depend on initial dispersions.

For an assessment it is necessary to use all available data, and, besides, additional difficulty consists in need of estimation of start state. We will construct an assessment which is "current", and doesn't use any assessment of a status. For this purpose it is necessary to assume that the following "a definability condition" is satisfied [8]:

$$
\begin{aligned}
& \lim _{N \rightarrow \infty} \frac{1}{N} \sum_{1}^{N} K_{n}^{*} C_{n}^{*} C K_{n} \\
& K_{n}=\sum_{0}^{n-1} A^{j} \tilde{U}_{n-1-j} ; \mathrm{U}-\text { input sequence of } \mathrm{p} \times 1 ;
\end{aligned}
$$

A and $\mathrm{C}-$ known matrixes $\mathrm{n} \times \mathrm{n}$ and $\mathrm{m} \times \mathrm{n}$.

The definability condition (criterion of identifiability) is that the limit (2) exists and is nondegenerate.

The definability condition also superimposes restriction on input sequence, that is in case of execution of a condition of definability (and stability of a matrix of model) the covariance matrix of an error aims at zero in case of $n$

Computation of criterion of identifiability by this technique assumes difficult mathematical computation therefore use of criterion in practical applications isn't possible.

The method of determination of identifiability [9] assumes introduction of other restrictions.

We will consider some most important types of restrictions and we will give criteria of identifiability.

Prior linear homogeneous functions are entered, each of which depends only from coefficients of a matrix of model which can be single-digit regenerated on a matrix of a reduced form [9].

The necessary condition of identifiability of a certain equation which can is provided formulated as follows: the number of the exogenetic variables excluded from the equation of $\alpha_{1}[\mathrm{~W} \Phi]=0$ system shall be at least number of the endogenous variables participating in it, reduced by unit.

$\mathrm{W}$ - the matrix of the order $(\mathrm{G}+\mathrm{K}) \mathrm{xK}$ having $\mathrm{K}$ rank; $\Phi$ - the matrix from $\mathrm{G}+\mathrm{K}$ of lines having so many columns, how many restrictions. Elements of a vector of $\alpha_{1}$ are the decision of system of equations of $\alpha_{1}[\mathrm{~W} \Phi]=0$.

The formulated necessary conditions (so-called rules of an order) owing to the simplicity are the very useful in case of a solution of the problem of identifiability as in case of creation of model they allow to reveal not identified equations directly. However these conditions can be far from 
sufficient. The necessary and sufficient condition isn't suitable for check of identifiability of model as requires creation of a complex array of a reduced form. Nevertheless it is possible to derive criterion of identifiability from it and in terms of the structural form (the rule of a rank).

This method of determination of identifiability of model parameters is suitable rather for the theoretical abstract.

Thus, some methods of determination of such property of matrixes of models as an identifiability level are probed. The provided criteria of a level of identifiability don't allow to carry out comparing of quality of identification of separate parameters of matrixes of models, are inconvenient in practical application therefore it is necessary to develop the universal criterion which isn't requiring difficult computation.

\section{Method of computation of a level of identifiability of parameters on the basis of scalar approach}

A lack of the considered methods of determination of a level of identifiability is need of carrying out difficult computation and absence of numerical evaluation of a level of identifiability of a specific matrix parameter of model.

We will carry out synthesis of criterion of a level of identifiability on the basis of scalar approach [10].

We will consider a mathematical model of the researched dynamic object which has the following appearance:

$$
x_{k}=\Phi x_{k-1}+w_{k-1}
$$

where $x_{k-1}-$ state vector; $w_{k-1}-$ a vector of input noise which is the discrete analog of white Gaussian noise with zero mathematical expectation; $\Phi-(\mathrm{n} \times \mathrm{m})$ - a model matrix.

The part of state vector is measured:

$$
z_{k}=H x_{k}+v_{k}
$$

where $z_{k}-\mathrm{a}$ m-vector of measurements; $H-(\mathrm{m} \times \mathrm{n})-$ a matrix of measurements; $v_{k}-\mathrm{a}$ m-vector of measuring noise which is the discrete analog of white Gaussian noise with zero mathematical expectation, and by $v$ and $w$ aren't correlated among themselves, i.e. $M\left[v_{j} w_{k}{ }^{T}\right]=0$.

The task of estimation of unknown constant matrix parameters of object $\Phi$ is set.

According to scalar approach the vector of the given measurements has an appearance [10]:

$$
\begin{aligned}
& z_{n+1}=H \Phi^{n}\left[\begin{array}{c}
H \\
H \Phi \\
\ldots \\
\ldots \\
H \Phi^{n-1}
\end{array}\right]^{-1}\left[\begin{array}{c}
z_{1} \\
z_{2} \\
\ldots \\
\ldots \\
z_{n}
\end{array}\right]-H \Phi^{n}\left[\begin{array}{c}
H \\
H \Phi \\
\ldots \\
\ldots \\
H \Phi^{n-1}
\end{array}\right]^{-1}\left[\begin{array}{c}
v_{1} \\
H w_{1} \\
H \Phi w_{1}+H w_{2}+v_{3} \\
\ldots \\
H \Phi^{n-2} w_{1}+\ldots+v_{n}
\end{array}\right]+ \\
& +H \Phi^{n-1} w_{1}+H \Phi^{n-2} w_{2}+\ldots+H w_{n}+v_{n+1}
\end{aligned}
$$

The equation for a vector of unknown parameters in the scalar form will have the following appearance:

$$
\begin{aligned}
& a_{1}=f_{1}\left(z_{1}, \ldots, z_{2 n}\right)+\mathbf{v}_{1}^{00} \\
& a_{2}=f_{2}\left(z_{1}, \ldots, z_{2 n}\right)+\mathbf{v}_{2}^{00} \\
& \ldots \ldots \ldots \ldots \ldots \ldots \ldots \ldots \ldots . . . \\
& a_{n}=f_{n}\left(z_{1}, \ldots, z_{2 n}\right)+\mathbf{v}_{n}^{00}
\end{aligned}
$$

By means of the equations (6) measurement of matrix parameters of object is carried out. Accuracy of determination of parameters depends on intensity of the given measuring noise. Value of the given measuring noise can reach the considerable values that leads to doubtful determination of parameters. Therefore for determination of unknown parameters it is expedient to use smoothing algorithms of measuring noise and algorithms of estimation $[3,8,10]$. As algorithms of estimation it is possible to use the well-known filter of Kallman [8,11]

As the parameters a1, a2,.., are constant an on a problem definition, for each ai parameter it is possible to write the assessment equation in a look. 


$$
\hat{a}_{1}^{i}=\hat{a}_{0}^{i}+k_{1}^{i}\left[f_{i}\left(z_{1}, \ldots, z_{2 n}\right)-\hat{a}_{0}^{i}\right]
$$

Where $\hat{a}_{1}^{i}, \hat{a}_{0}^{i}$ - parameter an $\mathrm{i}$ estimates in the first and in zero timepoints respectively; $\mathrm{k}_{1}^{\mathrm{i}}-$ optimum gain amount of the filter.

The optimum gain amount is defined from the following equation:

$$
k_{k}^{i}=\frac{p_{0}^{i}}{k p_{0}^{i}+r_{k}^{i}}
$$

Here $r_{k}^{i}=M\left[\left(v^{00}\right)^{2}\right]$ - dispersion of the given measuring noise of $v^{00}$.

This dispersion is the unknown even in that case when covariance matrixes of the initial input and measuring noise of $M\left[w_{k} w_{k}^{T}\right], M\left[\left(v_{k}\right)^{2}\right]$ are known. Value of the given noise of $v^{00}$ depends on unknown matrix parameters $\Phi$. For this reason it is necessary to use the adaptive algorithms of estimation for a case of absence of prior information on dispersion of measuring noise $[10,11,12]$. In this adaptive algorithm the assessment of dispersion of measuring noise by means of expression of a look is carried out:

$$
\hat{r}_{k}^{i}=\frac{1}{2} \sqrt{\left\{p_{0}^{1} k-M\left[\left(v_{k}^{i}\right)^{2}\right]\right\}^{2}+4 M\left[\left(v_{k}^{i}\right)^{2}\right] p_{0}(k-1)}-\frac{1}{2}\left\{p_{0}^{i} k-M\left[\left(v_{k}^{i}\right)^{2}\right]\right\}
$$

Measuring noise of $v^{00}$ is the discrete color noise even then, when the initial measuring noise of $v$ - a white noise. For color measuring noise it is possible to use the same algorithm of estimation [3], as for white measuring noise, but thus the algorithm of estimation becomes suboptimal.

Offered in [10] algorithm of identification actually realizes estimation of a vector $\left[\begin{array}{llll}a_{1} & \ldots & a_{n}\end{array}\right]^{T}$ which is connected to required matrix parameters $\Phi$ equation.

In case of the solution of the task of identification at the first stage it is necessary to clarify basic possibility of determination of matrix parameters of object, differently identifiability of parameters. In a problem definition of the identification offered in [10] identifiability of parameters can be defined by criterion of observability.

The system (3), (4) is called completely observable on time interval $\left[0, t_{1}\right]$ if the state vector $\mathrm{x}_{0}$ ) can be determined by a known vector of measurements of $\mathrm{z}(\mathrm{t})$. Check of observability can be realized, having used criterion of full observability of Kallman [3,8,11]. The system is completely observable if the rank of a matrix of a nablyukdayemost is equal to a system $n$ order. i.e. if measurements of $\mathrm{z}(\mathrm{t})$ contain sufficient information for determination $\mathrm{x}\left(\mathrm{t}_{0}\right)$. The matrix of observability of $\mathrm{S}$ has an appearance:

$$
S=\left[\begin{array}{c}
H \\
H \Phi \\
\cdot \\
H \Phi^{n-1}
\end{array}\right]
$$

If the rank of a matrix of observability is less than order of system, on measurements of $z(t)$ it is possible to evaluate only part of state vector $\mathrm{x}(\mathrm{t})$.

\section{Criterion of a level of identifiability}

In practical applications it is often necessary to know a level of identifiability of parameters, i.e. qualitative characteristics of the identified parameters: obtainable accuracy of identification of parameters and time for which it is possible to realize identification of parameter with the given accuracy.

It is possible to judge an identifiability measure for two characteristics: accuracy of identification and time of convergence. The criterion by which the level of identifiability is determined has an appearance:

$$
\gamma^{i}=\frac{P_{o}^{i} r}{P_{o} r^{*_{i}}}
$$

where P0 - dispersion of starting value directly the measured component of a vector of the identified parameters a. 
In a case when it isn't possible to define precisely starting value of dispersion of an error of identification, it is possible to use criterion of a level of identifiability of a look:

$$
\gamma^{i}=\frac{M\left[\left(a^{i}\right)^{2}\right] r}{M\left[\left(z^{i}\right)^{2}\right] r^{*_{i}}}
$$

Here $M\left[\left(a^{i}\right)^{2}\right]$ - dispersion of a component of a vector of parameters an arbitrary $\mathrm{i}$;

$M\left[\left(z^{i}\right)^{2}\right]$ - dispersion directly the measured component of a vector of parameters;

$\mathrm{r}=\mathrm{M}\left[\mathrm{v}^{2}\right]-$ dispersion of the initial measuring noise;

$r^{*_{i}}{ }_{k}=\frac{1}{2} \sqrt{\left\{p_{0}^{1} k-M\left[\left(v_{k}^{i}\right)^{2}\right]\right\}^{2}+4 M\left[\left(v_{k}^{i}\right)^{2}\right] p_{0}(k-1)}-\frac{1}{2}\left\{p_{0}^{i} k-M\left[\left(v_{k}^{i}\right)^{2}\right]\right\}-$ dispersion of the given measuring noise [10].

In criteria of a level of identifiability (11) and (12) measure of quality of identification the scalar is. This feature favourably distinguishes the offered criteria from known as allows to carry out comparing of levels of identifiability of components of vectors of parameters of matrixes of different objects. A measure of a level of observability is the scalar.

The developed formalized dependences can be used for determination of a level of identifiability of matrix parameters $\Phi$.

\section{Mathematical modeling}

It is possible to define quality of identification of concrete parameters of a matrix of model of dynamic object by the offered criterion. It is possible to judge quality of identification or efficiency of identification according to two characteristics: accuracy and time of identification. The most achievable accuracy of identification and necessary time before achievement of the set identification accuracy means.

We will consider known models of errors of INS [12,15] as an example. We will define degree of identifiability of the parameter of a matrix containing $\mathrm{g}$ - gravitational acceleration. In the course of flight of $g$ changes at change of height of flight. Therefore aprioristic value of $9,8 \mathrm{~m} / \mathrm{c}^{2}(588$ $\mathrm{m} / \mathrm{min}^{2}$ ) is required to be specified at change of height of flight of aircraft.

At first we will consider model of one horizontal INS [15] information channel. Degree of identifiability of $\mathrm{g} \gamma=0,001$. Then we investigate model of three INS channels. This model includes cross communications between information channels [12]. In this case $\gamma=0,003$. According to the offered criterion efficiency of identification of $g$ will be higher in case of use of more detailed model. This circumstance is explained by existence of additional communications of the identified parameter with direct measurements.

This result is confirmed by results of mathematical modeling.

Results of mathematical modeling of scalar algorithm of identification [15] are presented on fig. 1 .

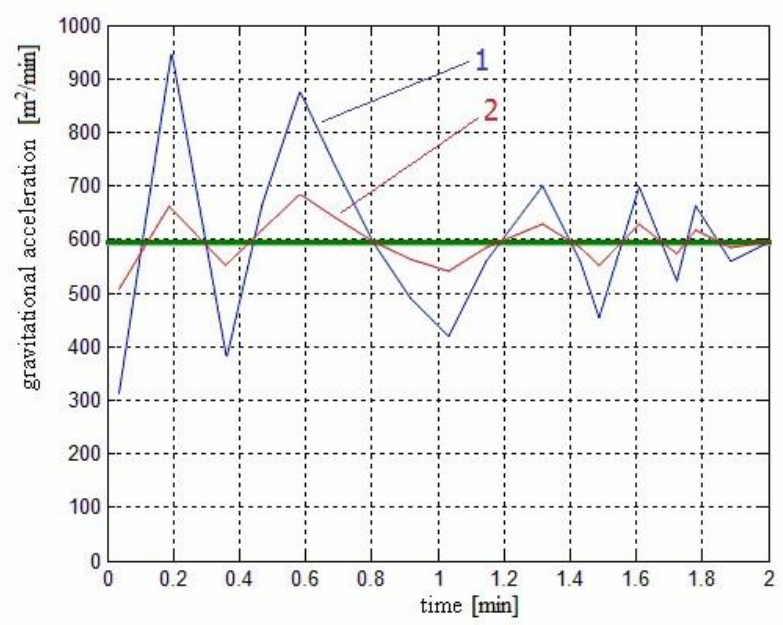

Fig.1: Results of scalar algorithm of identification 
In fig. 1 the following designations are entered: 1 assessment of parameter $\mathrm{g}$ by means of scalar algorithm of identification with use of model of one INS channel, 2 assessment of parameter $\mathrm{g}$ when using model of three INS channels. Apparently from schedules identification accuracy when using model of errors of three INS channels is $12 \%$ higher, than identification accuracy when using model of errors of one INS channel.

Thus, the offered criterion makes clear physical sense, differs in simplicity and is convenient in practical application.

\section{Conclusions}

The developed numerical criterion of a level of identifiability of specific matrix parameters of model of a dynamic object differs in universality and allows to calculate quality of identification of parameters in the form of a scalar.

The criterion differs in simplicity and is convenient in practical applications.

\section{References}

[1] Eykhoff P. Bases of identification of management systems. - M.: 1975

[2] Bryson, Huo of Yu Shi. Application-oriented theory of optimum control. - M.: World, 1972

[3] Kuzovkov N. T., Karabanov S.V., Salychev O. S. The continuous and discrete management systems and methods of identification. - M.: Mechanical engineering, 1978

[4] Neusypin K.A., Proletarian A.V., Tsibizova T.Yu. Management systems aircraft and algorithms of information processing. - M, MGOU, 2006

[5] Ivakhnenko A.G., Müller Y.Ya. Self-organization of predictive models. - Kiev, Technique, 1985

[6] Yemelyanov V. V., Kureychik V. V., Kureychik V. M. Theory and practice of evolutionary simulation. - M: Fizmatlit, 2003

[7] Balonin N. A. Theorems of identifiability. - SPb.: 2010

[8] Balakrishnan A.V. Theory of filtering Kallman: the lane with English - M.: World, 1988

[9] Ayvazyan S. A., Enyukov I. S., Meshalkin L. D. Application-oriented statistics: Research of dependences: M.: Finance and statistics, 1985

[10] Salychev O. S. Scalar estimation of multivariate dynamic systems. - M.: Mechanical engineering, 1987

[11]Neusypin K.A. The modern systems and methods of induction, navigation and control of aircraft. - M, Prod. MGOU, 2009. - 500s.

[12]Proletarsky A.V., Neusipin K.A. Adaptive filtering for navigation systems of robot-aerocraft. Science\& military, V.5., 2010. - pp.75-80.

[13]Neusipin K.A., Ke Fang. Research Progress of Intelligent Control Systems of Aerokrafts. Acta Armamentarii. Vol. 7, Jul. 2010. Print - ISSN 10001093.

[14]Fang Ke, A. Proletarsky, K. Neusipin. Selection of Measured Signals in the Navigation Measuring Complex Journal of Measurement Science and Instrumentation. December, 2011.04, v.02, c. 346-348.

[15]Proletarsky A., Neusipin K. Reserch scalar filtering algorithm with selforganization method for modeling control system. Science \& military, volume 5, № 2, 2010.

[16]Neusipin K.A., Ke Fang. The reasearch on modeling algorithm using self-organization method in aerocraft intelligent control system. Jornal of projectiles rocket missiles and guidance. Cep.30, №5, oct. 2010.,p.39-42. 